\title{
A INTERFERÊNCIA DOS TRANSTORNOS DE PERSONALIDADE NO TRATAMENTO DO TRANSTORNO DE PÂNICO: UMA ABORDAGEM COGNITIVO-COMPORTAMENTAL
}

\author{
Mariana Andrade Breschi Graduação em Psicologia pela Escola Bahiana \\ de Medicina e Saúde Pública(2011). \\ Atualmente é Pesquisadora da Escola Bahiana \\ de Medicina e Saúde Pública e psicóloga \\ clínica. Integrante do Dept. psicopedagógico \\ do Colégio Módulo. \\ Martha Moreira Cavalcante Castro \\ Doutora em Medicina e Saúde pela UFBA \\ Especialista em Terapia Cognitivo - \\ comportamental. Professora adjunta da \\ graduação e pós - graduação da Escola \\ Bahiana de Medicina e Saúde Pública e \\ Professora Adjunta Universidade Federal da \\ Bahia.
}

\begin{abstract}
Resumo
A ansiedade é uma emoção inerente ao ser humano, presente em todas as pessoas, fazendo parte do processo de adaptação ao meio em que vivem. A ansiedade, quando experimentada excessivamente, pode se tornar patológica, apresentando-se como um transtorno. Dentre os transtornos ansiosos, o transtorno de pânico é definido pela ocorrência de ataques recorrentes de ansiedade, os quais surgem subitamente sem nenhum motivo desencadeador aparente. Estudos apontam que a TCC é eficaz no tratamento do transtorno de pânico. Entretanto, quando há comorbidades associadas - a saber, transtornos de personalidade - as pesquisas mostram resultados menos significativos da intervenção cognitivo-comportamental para esse tipo de associação.
\end{abstract}

Palavras-chave: Transtorno de Pânico; Transtorno de personalidade; Terapia cognitivo comportamental.

\begin{abstract}
Anxiety is an emotion inherent in human beings, present in all people, being part of the process of adaptation to the environment in which they live. The anxiety, when overly experienced, may become pathological, presenting itself as a disorder. Among the anxiety disorders, panic disorder is defined by the occurrence of recurrent attacks of anxiety, which suddenly appears for no reason apparent trigger. Studies show that CBT is effective in the treatment of panic disorder. However, when there are associated comorbidities - namely, personality disorders the researches show less significant results of cognitive-behavioral intervention for this type of association.
\end{abstract}

Keywords: Panic disorder; Personality disorder; Cognitive behavioral therapy.

A ansiedade é uma emoção inerente ao ser humano. Todas as pessoas sentem a ansiedade em algum grau, todos os dias, podendo ser definida como um conjunto de sintomas, comportamentos ou sensações fisiológicas de tensão muscular, inquietação, batimentos cardíacos acelerados, causando certo desconforto no corpo. Entretanto, mesmo não sendo muito agradável, ela se manifesta quase que em todos os momentos importantes da vida do indivíduo. $^{(1)}$

De acordo com a categorização do Manual Diagnóstico e Estatístico: DSM-IV-TR, ${ }^{(2)}$ o transtorno de pânico é classificado dentro da categoria dos transtornos de ansiedade e seus principais sintomas são: sudorese, taquicardia, tremores, náuseas, parestesias, sensação de 
Breschi, Castro. A interferência dos transtornos de personalidade no tratamento do transtorno de pânico: uma abordagem cognitivocomportamental

asfixia, falta de ar, sensação de desmaio, despersonalização, desrealização, ondas de frio ou calor, medo de enlouquecer e sensação de morte iminente. Esses ataques são recorrentes e surgem repentinamente, sem nenhum evento desencadeador aparente e, logo após experimentar o primeiro ataque de pânico, o indivíduo desenvolve uma preocupação intensa com a ocorrência de um novo episódio.

Castillo $^{(3)}$ afirmam que, avaliando o medo e a ansiedade, é possível identificar se estes são patológicos ou não. Dentre os transtornos de ansiedade, o transtorno de pânico vem sido estudado intensamente nos últimos anos, havendo avanços em seu diagnóstico e tratamento. Existem duas modalidades de tratamento para o transtorno do pânico, sendo elas a psicoterapia individual ou em grupo e o tratamento farmacológico.

Yano, Meyer e Tung ${ }^{(4)}$ demonstraram que a terapia cognitivo-comportamental (TCC) tem experimentos empíricos, se mostrando eficaz para o tratamento dos transtornos ansiosos, dentre os quais o transtorno do pânico. A TCC no tratamento do transtorno do pânico se baseia no modelo de Barlow e Clark, criado no ano de 1986, o qual sugere que o indivíduo reage a um sinal de perigo imaginário, tendo sensações corporais subseqüentes à interpretação catastrófica desse sinal.

A TCC utiliza, como um dos elementos no tratamento, a psicoeducação para o transtorno de pânico e ansiedade, e a reestruturação cognitiva, cujo objetivo é ensinar ao paciente quais pensamentos e interpretações ocorrem no momento em que tem os sintomas de ansiedade. A intervenção comportamental também é utilizada para o manejo dos ataques de pânico, principalmente propondo a exposição do paciente às situações temidas. Técnicas como respiração diafragmática também são utilizadas para o controle do equilíbrio do sistema nervoso autônomo, com bons resultados. No final do tratamento, é realizada a prevenção de recaída para ensinar ao paciente a identificar situações potencialmente ansiogênicas a fim de que, a partir daí, ele possa utilizar as técnicas aprendidas na terapia para o adequado controle da sua ansiedade. ${ }^{(5)}$

$\operatorname{Ramos}^{(6)}$ afirma que há hipóteses neuroquímicas para o transtorno de pânico sendo levantadas a partir de experimentos realizados com testagem de medicações que tem algum efeito nos sintomas que caracterizam um ataque de pânico. Desta forma, as hipóteses mais exploradas relacionam o transtorno de pânico a neurotransmissores, como serotonina e noradrenalina. De acordo com Yano, Meyer e Tung, ${ }^{(4)}$ existe uma atividade noradrenérgica excessiva enquanto a transmissão de serotonina é deficitária. Tal observação foi feita a partir de estudos realizados com antidrepressivos, que aumentam a serotonina nas fendas sinápticas, 
Breschi, Castro. A interferência dos transtornos de personalidade no tratamento do transtorno de pânico: uma abordagem cognitivocomportamental

constatando-se uma melhora dos sintomas neste paciente no intervalo de 21 dias, aproximadamente.

De acordo com American Psychiatric Association, ${ }^{(2)}$ estudos epidemiológicos no mundo encontraram uma prevalência durante a vida toda de $1,5 \%$ e $3,5 \%$, e ao longo do ano, de $1 \%$ e $2 \%$ de transtorno de pânico. Um estudo realizado nos Estados Unidos mostrou que $5 \%$ da população estudada apresentaram transtorno de pânico ao longo da vida. Não existem estudos de prevalência do transtorno de pânico no Brasil, porém um estudo realizado em São Paulo apresentou uma estimativa da presença do transtorno de pânico de 1,6\% ao longo da vida e $1 \%$ no ano, concluindo que aparentemente não existe uma variabilidade de prevalência entre o Brasil e os estudos que abragem todas as populações. O transtorno de pânico ocorre duas vezes mais em mulheres do que em homens, o seu início tem uma variabilidade grande, mas o mais comum é ocorrer a partir do final da adolescência e entre os 20 e os 30 anos de idade. $^{(7)}$

O desenho de estudo para a realização do trabalho foi uma revisão tradicional da literatura, que teve como base de dados eletrônicos o Medline, PUBMED, Scielo, Bireme, Periódicos Capes, nos idiomas português e inglês, e livros especializados. Foram utilizadas palavras-chaves como panic disorder, personality disorder, cognitive-behavioral therapy, transtorno de personalidade, transtorno de pânico e terapia cognitivo-comportamental, para a busca dos artigos.

Essa revisão tem como objetivo discutir qual a implicação no tratamento cognitivocomportamental do transtorno de pânico para pessoas que apresentam comorbidades com o transtorno de personalidade.

Salum, Blaya e Manfro ${ }^{(7)}$ afirmam que uma alta porcentagem $(83 \%)$ dos pacientes com diagnóstico de transtorno do pânico possuem comorbidades psiquiátricas de Eixo I. Outros estudos também comprovam a existência de transtornos de personalidade associados a portadores de transtorno do pânico, o que pode implicar na resposta ao tratamento e na prática clínica. ${ }^{(8)}$ De acordo com Savoia, Zuccolo e Corchs, ${ }^{(9)}$ a relação entre os transtornos de personalidade e transtornos de Eixo I é estreita e complexa.

Segundo Mennin e Heimberg, ${ }^{(10)}$ comorbidades associadas ao transtorno do pânico apontam controvérsias ao tratamento dessa patologia. Slaap e Boer ${ }^{(11)}$ afirmam que os transtornos de personalidade, ou até mesmo traços de personalidade, são possivelmente um dos principais fatores preditores de não resposta ao tratamento para o transtorno de pânico.

Revista Psicologia, Diversidade e Saúde, Salvador. 2013 Abr;1(1):21-28. 
Breschi, Castro. A interferência dos transtornos de personalidade no tratamento do transtorno de pânico: uma abordagem cognitivocomportamental

Porém, um estudo realizado concluiu que o transtorno de personalidade não interfere nas respostas do tratamento do transtorno de pânico. ${ }^{(12)}$ Outro estudo realizado por Prasko ${ }^{(13)}$ mostrou que o tratamento cognitivo-comportamental é mais eficaz para os sintomas de ansiedade em pacientes com transtorno de pânico apenas; porém, quando associado ao transtorno de personalidade, o mesmo tratamento também apresenta resultados satisfatórios. Gude e Hoffart ${ }^{(14)}$ demonstraram em um estudo de corte que pacientes com transtorno de pânico, comórbidos com transtorno de personalidade do grupo $\mathrm{C}$, apresentam resultados eficazes da TCC para os sintomas do transtorno de pânico.

A TCC fundamenta-se a partir do modelo cognitivo, se baseando na idéia de que os comportamentos e emoções das pessoas são influenciados pela percepção dos eventos e o modo pelo qual interpretam determinada situação. Os pensamentos automáticos surgem de forma repentina, são rápidos e breves. Dessa forma, é comum que o individuo os aceite como verdadeiros, não criticamente, devido à rapidez em que acontecem. ${ }^{(15)}$

Além de comorbidades de Eixo I com o transtorno de pânico, citado acima, é possível que alguns indivíduos que apresentam o transtorno de pânico também possam apresentar traços ou diagnóstico de transtorno de personalidade (Eixo II). Um estudo realizado para verificar a prevalência e co-ocorrência de transtornos de personalidade com indivíduos portadores de transtorno de ansiedade concluiu que essa relação é positiva e significativa. Os transtornos de personalidade (TP) esquiva e dependente foram mais fortemente associados aos transtornos de ansiedade, sendo que os TP paranóide, esquizóide, histriônico e anti-social foram mais relacionados ao transtorno de pânico. ${ }^{(16)}$

Estudos revelam que transtornos de personalidade aumentam a gravidade do transtorno do pânico. ${ }^{(17)}$

De acordo com a American Psychiatric Association, ${ }^{(2)}$ os transtornos de personalidade se caracterizam por apresentar uma repetição de condutas que não seguem o padrão da cultura ao qual o indivíduo está inserido. "Um Transtorno da Personalidade é um padrão persistente de vivência íntima ou comportamento que se desvia acentuadamente das expectativas da cultura do indivíduo, é invasivo e inflexível". Esse padrão traz perdas e sofrimentos para o sujeito, se inicia na adolescência ou início da idade adulta, persistindo ao longo da vida do indivíduo, e torna-se uma forma de funcionamento fixo da personalidade. Este manual aponta que são dez os tipos de transtorno de personalidade, divididos em três grupos distintos, a saber: Grupo A - TP paranóide, esquizotípica, esquizóide; Grupo B - anti-social, borderline, histriônica, narcisista; e Grupo C - evitativo, dependente e obsessivo-compulsivo. 
Breschi, Castro. A interferência dos transtornos de personalidade no tratamento do transtorno de pânico: uma abordagem cognitivocomportamental

A prevalência dos transtornos de personalidade tem sido pouco estudada. Pesquisas com metodologia adequada e que utilizam um número representativo de pessoas para a população em geral mostraram que a prevalência dos transtornos de personalidade se aproxima de $10 \%$ para a população mundial, incluindo todos os grupos caracterizados pelo DSM-IV. Estudos envolvendo correlação entre gênero e transtornos de personalidade apontam uma associação do sexo masculino com todos os subtipos de transtorno de personalidade, principalmente no que se refere ao diagnóstico do Grupo A. ${ }^{(18)}$

A TCC percebe o transtorno da personalidade a partir da história de vida do sujeito e como esse estabeleceu os seus padrões de pensamento, sentimento e comportamento. Os esquemas, ou seja, as crenças do sujeito são estruturadas e servem como referência para interpretação das experiências vividas. Dessa forma, as crenças do indivíduo interferem diretamente nos pensamentos, afetos e comportamentos. As percepções das aprendizagens podem ser modificadas a depender dos esquemas da pessoa quando estes se tornam rígidos, ou seja, são pouco afetados pelas experiências vividas e podem sustentar o surgimento de transtornos de personalidade ou esquemas disfuncionais. ${ }^{(19)}$

Pacientes com transtorno de pânico geralmente apresentam estas condições comórbidas, mas, em sua maior parte, estudos sobre tratamento cognitivo-comportamental têm prestado pouca atenção a eles. A maioria dos estudos sobre os efeitos da co-morbidade no tratamento se preocupa com resultados farmacológicos. No entanto, a literatura assinala que há um efeito dos transtornos de personalidade no resultado das intervenções cognitivocomportamental em pessoas que apresentam o transtorno do pânico. ${ }^{(10)}$

Um estudo de pessoas com transtorno de pânico comórbidos com transtornos de personalidade demonstra que, ao serem submetidos à terapia psicodinâmica e psicoterapia cognitivo-comportamental focada em esquemas, apresentaram como resultado uma melhora significativa dos pacientes submetidos à terapia cognitiva em relação aos pacientes submetidos à terapia psicodinâmica, no que diz respeito a problemas interpessoais. Desse modo, concluiu que a TCC é um dos tratamentos mais indicados para pessoas com esses dois diagnósticos comórbidos. ${ }^{(14)}$

Entretanto, a literatura não é unânime quando demonstra eficácia da TCC em pacientes que apresentam diagnósticos superpostos de transtorno de pânico e transtorno de personalidade. Estudos mostram que existem uma resposta mais pobre ao tratamento cognitivo-comportamental quando está presente a associação transtorno de personalidade e 
Breschi, Castro. A interferência dos transtornos de personalidade no tratamento do transtorno de pânico: uma abordagem cognitivocomportamental

transtorno de pânico e sugerem que esses pacientes não são resistentes ao tratamento, entretanto, precisam de um acompanhamento mais duradouro. ${ }^{(20,21)}$

Conclui-se, dessa forma, que a TCC exerce uma efetividade maior em pacientes que apresentam o transtorno de pânico sem comorbidades. Entretanto, observou-se que pacientes com transtorno de personalidade associado ao transtorno de pânico, apesar de estudos apontarem para uma resposta mais pobre desses pacientes, alcançam uma melhora significativa após o tratamento cognitivo-comportamental, principalmente no que diz respeito à redução dos sintomas ansiosos, sugerindo uma intervenção mais prolongada.

Considerando a complexidade do tema analisado, sugere-se que estudos continuem sendo realizados para possibilitar o esclarecimento a respeito da efetividade e contribuições da TCC em pacientes que apresentam transtorno de pânico associado a transtornos de personalidade, bem como de que forma pacientes e profissionais podem se posicionar diante do processo terapêutico e desenvolver estratégias de manejo da saúde mental do indivíduo.

\section{REFERÊNCIAS}

1 Craske MG, Barlow DH. Transtorno de pânico e agorafobia. In: Barlow DH. Manual clínico dos transtornos psicológicos. Porto alegre: Artmed; 2009.

2 AMERICAN PSYCHIATRIC ASSOCIATION. Manual diagnóstico e estatístico de transtornos mentais: DSM-IV-TR. 4를 ed. Porto Alegre: Artmed; 2002.

3 Castillo ARGL. Recodo R, Asbahr FR, Manfro GG.Transtornos de ansiedade Rev Bras Psiquiatr, 2000;22(Supl II):20-3.

4 Yano Y, Meyer SB, Tung TC. Modelos de tratamento para o transtorno do pânico. Estudos de psicologia [internet]. 2003 Set/Dez;20(3). [acesso em 2011 Maio 13]Disponível em: $<$ http://www.scielo.br/scielo.php?script=sci_arttext\&pid=S0103$166 X 2003000300009 \& \operatorname{lng}=\mathrm{en} \& \mathrm{nrm}=\mathrm{iso}>$.

5 Freedman S, Adessky R. Cognitive behavior therapy for panic disorder. Isr. J Psychiatry Relat Sci. 2009;46(4):251-6.

6 Ramos RT. As bases biológicas do transtorno de pânico. Rev de Psiquiat Clín. 2001;28(1): 9-11

7 Salum GA, Blaya C, Manfro GG. Transtorno do pânico. Rev Psiquiatr Rio Gd Sul. [internet]. 2009;31(2) [acesso em Maio 13] Disponível em: http://www.scielo.br/scielo.php?script=sci_arttext\&pid=S0101$81082009000200002 \& \operatorname{lng}=$ en $\& n r m=$ iso 
Breschi, Castro. A interferência dos transtornos de personalidade no tratamento do transtorno de pânico: uma abordagem cognitivocomportamental

8 Starcevic V, Latas M, Kolar D,Vucinic-Latas D, Bogogievic G, Milovanovic S. Cooccurrence of Axis I and Axis II disorders in female and male patients with panic disorder with agoraphobia. Compr Psychiatry. 2008 Nov/Dec;49(6):537- 43.

9 Savoia MG, Zuccolo, PF, Corchs F. Personalidade e transtornos de ansiedade In: Range B. Psicoterapias cognitivo-comportamentais:um dialogo com a psiquiatria. $2^{\mathrm{a}}$ ed. Porto Alegre: Artmed; 2011. p. 751-59

10 Mennin DS, Heimberg RG. The impact of comorbid mood and personality disorders in the cognitive-behavioral treatment ofpanic disorder. Clin Psychol Rev. 2000;20(3):339-57.

11 Slaap BR, Den Boer JA The prediction of non response to pharmacotherapy in panic disorder: a review. Depress Anxiety. 2001;14(2):112-22.

12 Massion AO, Dyck IR, Shea MT, Phillips KA, Warshaw MG, Keller MB. Personality disorders and time to remission in generalized anxiety disorder, social phobia, and panic disorder. Arch Gen Psychiatry. 2002 Mai;59(5):434-40.

13 Prasko J,Houbova P, Novak T, Zalesky R, Espa-Cervená K Paskova B, Vyskocilova J. Influence of personality disorder on the treatment of panic disorder-comparison study. Neuro Endocrinol Lett. 2005 Dez; 26(6): 6667-74

14 Gude T, Hoffart A. Change in interpersonal problems after cognitive agoraphobia and schema-focused therapy versus psychodynamic treatment as usual of inpatients with agoraphobia and Cluster C personality disorders. Scand J Psychol. 2008;49(1):195-99.

15 Greenberger D, Padesky C. A mente vencendo o humor: mude como você se sente, mudando o modo como você pensa. Porto Alegre: Artmed; 1999.

16 Grant BF, Hasin DS, Stinson FS, Dawson DA, Patricia Chou S, June Ruan W, Huang B. Co-occurrence of 12-month mood and anxiety disorders and personality disorders in the US: results from the national epidemiologic survey on alcohol and related conditions. J Psychiatri Res. 2005 Jan; 39(1):1-9.

17 Ozkan M, Altindag A. Comorbid personality disorders in subjects with panic disorder: do personality disorders increase clinical severity? Compre Psychiatry. $2005 \mathrm{Jan} / \mathrm{Fev}$;46(1): 206.

18 Peluso M, Andrade LHSG. Transtorno da personalidade: epidemiologia. In: Louza Neto M, Cordás TA. Transtornos de personalidade, Porto Alegre: Artemed; 2011. p. 25-38.

19 Vasques F. Abreu CN. Terapia cognitiva nos transtornos de personalidade. In: Louza Neto MR, Cordás, T. A. Transtornos de personalidade. Porto Alegre: Artemed; 2011. p. 309-21. 
Breschi, Castro. A interferência dos transtornos de personalidade no tratamento do transtorno de pânico: uma abordagem cognitivocomportamental

20 Berger P, Sachs G, Amering M, Holzinger A, Bankier B, Katschnig H. Personality disorder and social anxiety predict delayed response in drug and behavioral treatment of panic disorder. J Affec Disord. 2004;80(1):75-8.

21 Telch MJ, Kamphuis JH, Schmidt NB. The effects of comorbid personality disorders on cognitive behavioral treatment for panic disorder. J Psychiatric Res. 2011 Apr;45(4): 469-74. 